\title{
Identificación de bacterias patógenas oportunistas en útero de alpaca pre y poscópula
}

\author{
IDENTIFICATION OF OPPORTUNISTIC PATHOGENIC BACTERIA IN THE UTERUS OF ALPACA PRE- \\ AND POST-COPULA
}

\author{
Helen Dellepiane G. ${ }^{1}$, Siever Morales-Cauti ${ }^{2,3}$
}

\section{Resumen}

\begin{abstract}
El presente estudio tuvo como objetivo la identificación de bacterias presentes en la mucosa uterina de alpaca antes y después de la cópula. Se tomaron 237 muestras de mucosa uterina (177 antes y 60 después de la cópula). Para la toma de la muestra se utilizaron espéculos metálicos e hisopos protegidos para evitar contaminaciones de las muestras. Las muestras fueron conservadas y remitidas al laboratorio en tubos con medio de transporte Stuart a $4{ }^{\circ} \mathrm{C}$. Las muestras fueron sembradas en agar sangre y MacConkey a $37^{\circ} \mathrm{C}$ por $24 \mathrm{~h}$ y la identificación bacteriana se realizó a través de pruebas bioquímicas. Se identificaron 165 aislados bacterianos y 29 levaduras en el grupo precópula y 64 aislados bacterianos y 6 levaduras en el grupo poscópula. Las bacterias con mayor frecuencia fueron E. coli (24.9\%), Providencia stuartii (16.5\%), Shigella sp (16.5\%), Hafnia alvei (5.9\%), Serratia rubidae (5.9\%) y levaduras (14.8\%).
\end{abstract}

Palabras clave: alpaca; cópula; útero; bacterias

\section{Abstract}

The aim of the present study was to identify bacteria present in the uterine mucosa of alpaca before and after the copula. Samples of uterine mucosa were taken ( 177 before and 60 after copulation). For the collection of the sample, metallic speculum and protected

\footnotetext{
${ }^{1}$ Laboratorio de Microbiología, Facultad de Medicina Veterinaria y Zootecnia, Universidad Científica del Sur, Lima, Perú

${ }^{2}$ Laboratorio de Microbiología y Parasitología Veterinaria, Facultad de Medicina Veterinaria, Universidad Nacional Mayor de San Marcos, Lima Perú

${ }^{3}$ Email: sieverm@hotmail.com
}

Recibido: 14 de julio de 2017

Aceptado para publicación: 31 de diciembre de 2017 
swabs were used to avoid sample contamination. The samples were conserved and transported to the laboratory in tubes with Stuart transport medium at $4{ }^{\circ} \mathrm{C}$. Samples were cultured on blood agar and MacConkey agar at $37^{\circ} \mathrm{C}$ for $24 \mathrm{~h}$ and bacterial identification was performed through biochemical tests. A total of 165 bacterial isolates and 29 yeasts in the pre-copula group and 64 bacterial isolates and 6 yeasts in the post-copula group were found. The bacteria most frequently isolated were E. coli $(24.9 \%)$, Providencia stuartii (16.5\%), Shigella sp (16.5\%), Hafnia alvei (5.9\%), Serratia rubidae (5.9\%) and yeasts $(14.8 \%)$.

Key words: alpaca; copulation; uterus; bacteria

\section{INTRODUCCIÓN}

En el Perú se cuenta con 3685516 alpacas (Vicugna pacos), según el INEI (2012), perteneciendo más del $80 \%$ a las comunidades campesinas y a pequeños productores, y el $20 \%$ restante a medianos productores y empresas asociativas (FAO, 2005).

Durante los meses de diciembre y abril (época de lluvias y de mayor disponibilidad de pastos), los camélidos hembras presentan actividad sexual marcada y, es en ese momento, que manifiestan un celo continuo hasta la cópula (FAO, 2005). En esos meses se lleva a cabo el empadre controlado dentro del hato de alpacas para obtener un mayor porcentaje de preñez y número de crías (Cárdenas, 2013).

El lumen uterino es estéril, pero gérmenes del ambiente de los animales pueden colonizarlo durante el parto o en el tiempo inmediato a este (Sheldon, 2004; Foldi et al., 2006); además de las complicaciones posparto (placenta retenida) y la manipulación antihigiénica de los genitales (Tibary et al., 2001; Tibary, 2004). Asimismo, dada la copulación repetitiva en estas especies y considerando que la alpaca macho realiza un apareamiento agresivo que puede inducir una inflamación uterina severa (Tibary et al., 2001; Vaughan y Tibary, 2006); que sumado a posibles fallas en los mecanismos de defensa uterino, pueden conllevar al establecimiento de la infección (Tibary et al., 2006).
En el útero de camellos (Camelus dromedarius) aparentemente sanos se ha reportado la presencia de Streptococcus pyogenes, Staphylococcus aureus, Escherichia coli, Corynebacterium spp, y a nivel vaginal a Streptococcus pyogenes, Staphylococcus aureus, E. coli, Proteus spp y Corynebacterium spp (Mshelia et al., 2014).

El pene llega a penetrar hasta el útero durante la cópula, depositando el semen en ambos cuernos uterinos mediante movimientos leves de empuje (Bravo, 1994). Este proceso está relacionado a enfermedades de transmisión sexual, inducidas después de la cópula con machos infectados, como se vio en equinos infectados por Pseudomona aeruginosa (Platt et al., 1977). Por otro lado, el eyaculado de un macho sano no contiene microorganismos, pero la contaminación puede ocurrir durante la colección del semen (Althouse et al., 2000), debido a la posición de sentado que presentan durante la cópula. Ghoneim et al. (2014) observaron contaminación por $E$. coli y bacterias de los géneros Staphylococcus, Bacillus, Streptococcus, Klebsiella, Shigella, Actinomyces y Corynebacterium en muestras de semen tomadas por electroeyaculación en camello.

Ante esto, el presente trabajo tuvo como objetivo identificar bacterias que puedan estar presentes en la mucosa uterina pre y poscópula en alpacas (Vicugna pacos) con $\mathrm{y} \sin$ antecedentes reproductivos. 


\section{Materiales y Métodos}

\section{Ubicación del Estudio}

La toma de muestras y su procesamiento se realizó entre enero y marzo de 2015. Los animales se encontraban en los distritos de Ninacaca y Huallay, provincia de Pasco, Perú. Ninacaca se encuentra a $4140 \mathrm{msnm}$ y los animales muestreados pertenecen a la Sociedad de Productores de Llamas y Alpacas (SPAR). El distrito de Huallay se encuentra a $45 \mathrm{~km}$ de la ciudad de Cerro de Pasco y a una altura de $4340 \mathrm{~m}$. Las muestras fueron procesadas en el Laboratorio de Microbiología Veterinaria de la Universidad Científica del Sur, en Lima.

\section{Población y Muestras}

Las muestras de contenido uterino fueron colectadas en alpacas Huacaya de color blanco, que se encontraban separadas en puntas o grupos de machos y hembras, donde solo se juntaban para el momento del apareamiento. Se trabajó con un grupo de alpacas hembras sin experiencia sexual (alrededor de un año), y otro grupo de hembras con experiencia sexual (más de dos años).

Las muestras fueron colectadas $30 \mathrm{mi}-$ nutos antes de la cópula, aproximadamente, y a las 24 horas del apareamiento. Se colectaron 29 muestras precópula y 20 muestras poscópula de las hembras sin experiencia sexual en Ninacaca y 148 muestras precópula y 40 muestras poscópula de hembras con experiencia sexual en Huallay. Las diferencias en el número de muestras pre y poscópula se debieron a que no todas las hembras muestreadas entraron a empadre.

Las muestras fueron colectadas con hisopos estériles (adaptación de la torunda de cultivo uterino, Minitube, 2017), los cuales contaron con un protector plástico estéril que se adaptó para poder realizar la toma de muestra evitando posibles contaminaciones. Antes de la toma de muestra se realizó una breve inspección para verificar que los animales estaban aparentemente sanos; además, se revisó la zona vulvar para observar posibles laceraciones o secreciones vaginales.

Para la toma de muestra se desinfectó la parte externa de la vulva con algodón impregnado con clorhexidina al $0.5 \%$. Luego se introdujo un espéculo de metal por la vulva, previamente desinfectado entre cada animal muestreado. El espéculo llevaba un gel no espermicida para facilitar su introducción y una linterna para ubicar la entrada del cérvix. Una vez introducido el hisopo en el útero, se presionó desde afuera para liberarlo de la funda protectora y poder tomar la muestra de la mucosa uterina. Luego de hacer el hisopado se retiró el hisopo hacia atrás para que quedara nuevamente dentro de la funda protectora, y una vez dentro de esta se retiraba el hisopo protegido del espéculo y luego el espéculo. Finalmente, el hisopo era retirado de la funda protectora y colocado hasta el tercio superior del medio de transporte Stuart para su posterior procesamiento. Las muestras se llevaron de inmediato al laboratorio para su procesamiento.

\section{Procesamiento de las Muestras}

Se utilizó agar McConkey y agar sangre para el aislamiento de bacilos gramnegativos y grampositivos de fácil desarrollo, aerobios y anaerobios facultativos, incluyendo bacterias de la familia Enterobacteriaceae. La siembra se hizo directamente a partir del hisopo y por agotamiento en forma estriada. Las muestras fueron incubadas a $37{ }^{\circ} \mathrm{C}$ durante $24 \mathrm{~h}$. Los hisopos fueron, además, inoculados en caldo Rappaport a $41{ }^{\circ} \mathrm{C}$ durante $24 \mathrm{~h}$ para el enriquecimiento de Salmonella spp y luego en Agar XLD a $37^{\circ} \mathrm{C}$ por $24 \mathrm{~h}$ (OIE, 2008).

Las colonias obtenidas fueron teñidas con coloración Gram. La identificación se hizo mediante pruebas bioquímicas apropiadas para cada tipo bacteriano (grampositivas o gramnegativas), empleando agar citrato de Simmons, prueba de ureasa, prueba en me- 
dio SIM (sulfidrilo, indol y movilidad), agar tres azúcares (TSI), lisina iron agar (LIA) y las pruebas de oxidasa, catalasa, manitol, y esculina (Koneman et al., 2006).

\section{Resultados}

Los aislamientos bacterianos más importantes fueron de Providencia stuartii (15.8 y $18.3 \%)$, Shigella $\mathrm{sp}(17.5$ y $13.3 \%)$, E. coli (26.0 y $21.7 \%)$, Serratia rubidae (6.2 y $5.0 \%)$, Hafnia alvei (5.1 y $8.3 \%$ ) y levaduras $(16.4$ y $10.0 \%)$ en muestras uterinas antes y después de la cópula, respectivamente (Cuadros 1 y 2).

\section{Discusión}

Se encontraron especies bacterianas de la familia Enterobacteriaceae como Escherichia coli (24.9\%), Serratia rubidae (5.9\%) Providencia alcalifaciens (2.5\%), Enterobacter aerogenes $(2.1 \%)$ y Edwarsiella tarda (1.3\%) en la mucosa uterina de alpacas de muestras colectadas antes y después de la cópula. Algunas de estas bacterias también han sido reconocidas como parte de la microbiota bacteriana de secreciones uterinas de vacas como $E$. coli y Proteus sp (Fernández y Dimoso, 1984). Así mismo, ha sido hallado Escherichia coli $(33.8 \%)$ y otras especies de coliformes $(18.6 \%)$ en útero de camellos y E. coli (57.6\%), Klebsiella spp (2.2\%) y Pseudomonas spp (1.1\%) en útero de vacas, indicando la posibilidad de contaminación tipo ambiental (Azawi y Taha, 2002; Ghanem et al., 2002), lo cual es posible en el presente estudio, debido a las características de manejo de la especie y el potencial contacto en cada uno de los momentos del proceso de la cópula. Estas bacterias se encuentran en el ambiente y es posible la contaminación bacteriana del pene en el macho y la vagina y vulva en las hembras al echarse en el suelo como ocurre en otras especies (Azawi y Taha, 2002; Ghanem et al., 2002).
Se identificaron bacterias grampositivas como Staphylococcus spp (3.5 y 1.4\%) en alpacas sin y con experiencia sexual previa en su vida reproductiva, respectivamente, previo a la cópula (Cuadro 1). En forma similar, en camellos con y sin descargas vaginales se reportó Staphylococcus epidermidis, $S$. aureus y $S$. pyogenes a nivel uterino, sin que hubiera relación entre los tipos de descargas vaginales y la presencia de bacterias en el útero. También se aisló Staphylococcus aureus en útero y vagina de camellos aparentemente sanos (Mshelia et al., 2014), lo cual sugiere que Staphylococcus spp puede presentarse en la mucosa uterina de alpacas aparentemente sanas antes de la cópula.

Adicionalmente se encontró a Bacillus spp (5.5\%) y Corynebacterium spp (0.8\%) en la mucosa uterina de las alpacas. Especies como $C$. diphtheroides y $B$. anthracoides han sido observadas en yeguas y vacas como microorganismos contaminantes y comensales (Ricketts, 1981), así como en el útero de dromedarios sanos (Camelus dromedarius) y con endometritis (Enany et al., 1990) y de camellos aparentemente sanos (Mshelia et al., 2014).

Fusobacterium spp solo se encontró en dos casos previos a la cópula. Esta bacteria forma parte de la flora normal del tracto genitourinario animal (Jang y Hirsh, 1994), en tanto que $F$. nucleatum ha sido aislado en prepucio de camélidos sudamericanos (CSA) machos (Jarvien y Kinyon, 2010).

Streptococcus pyogenes ha sido hallado en útero de camellos sin descarga vaginal y aparentemente sanos (Mshelia et al., 2014) y en el cérvix en diferentes periodos del ciclo estral de vacas clínicamente sanas se ha reportado la presencia de Streptococcus viridans (Fernández et al., 1984). En el presente estudio se encontró Streptococcus spp en útero de alpacas con previo contacto sexual antes $(2.7 \%)$ y después de la cópula $(5 \%)$ (cuadros 1 y 2), lo cual indicaría que puede 
Cuadro 1. Especies bacterianas aisladas de mucosa de útero de alpacas sin experiencia sexual $(n=29)$ y con experiencia sexual $(n=148)$ colectadas 30 minutos previo a la cópula

\begin{tabular}{lccccccc}
\hline \multirow{2}{*}{ Bacteria } & \multicolumn{2}{c}{$\begin{array}{c}\text { Sin } \\
\text { experiencia }\end{array}$} & \multicolumn{2}{c}{$\begin{array}{c}\text { Con } \\
\text { experiencia }\end{array}$} & & Total & \\
\cline { 2 - 8 } & $\%$ & $\pm \mathrm{IC}$ & $\%$ & $\pm \mathrm{IC}$ & $\%$ & $\pm \mathrm{IC}^{1}$ & $\mathrm{n}=177$ \\
\hline Bacillus $\mathrm{sp}$ & 3.4 & 0.05 & 6.8 & 0.03 & 6.2 & 0.03 & 11 \\
Citrobacter feundii & 3.4 & 0.05 & 0 & 0 & 0.6 & 0.01 & 1 \\
Corynebacterium sp & 3.4 & 0.05 & 0.7 & 0.01 & 0.6 & 0.01 & 1 \\
Edwarsiella tarda & 3.4 & 0.05 & 0.7 & 0.01 & 1.1 & 0.01 & 2 \\
Enterobacter aerogenes & 3.4 & 0.05 & 0.7 & 0.01 & 1.1 & 0.01 & 2 \\
Enterobacter clocacae & 6.9 & 0.07 & 0 & 0 & 1.1 & 0.01 & 2 \\
Escherichia coli & 37.9 & 0.13 & 23.7 & 0.05 & 26.0 & 0.05 & 46 \\
Fusobacterium sp & 0 & 0 & 1.4 & 0.01 & 1.1 & 0.01 & 2 \\
Hafnia alvei & 0 & 0 & 6.1 & 0.03 & 5.1 & 0.02 & 9 \\
Micrococcus sp & 0 & 0 & 2.7 & 0.02 & 2.3 & 0.02 & 4 \\
Proteus mirabilis & 3.4 & 0.05 & 0 & 0 & 0.6 & 0.01 & 1 \\
Providencia alcalifaciens & 6.9 & 0.07 & 0.7 & 0.01 & 1.7 & 0.01 & 3 \\
Providencia stuartii & 24.1 & 0.11 & 14.1 & 0.04 & 15.8 & 0.04 & 28 \\
Serratia liquefancies & 0 & 0 & 0.7 & 0.01 & 0.6 & 0.01 & 1 \\
Serratia rubidae & 3.4 & 0.05 & 6.8 & 0.03 & 6.2 & 0.03 & 11 \\
Shigella sp & 20.7 & 0.11 & 16.9 & 0.04 & 17.5 & 0.04 & 31 \\
Staphylococcus sp & 3.4 & 0.05 & 1.4 & 0.01 & 1.7 & 0.01 & 3 \\
Streptococcus sp & 0 & 0 & 2.7 & 0.02 & 2.3 & 0.02 & 4 \\
BNF ${ }^{2}$ & 0 & 0 & 2.0 & 0.02 & 1.7 & 0.01 & 3 \\
Levaduras & 0 & 0 & 19.6 & 0.05 & 16.4 & 0.04 & 29 \\
\hline
\end{tabular}

${ }^{1}$ Intervalo de confianza del 95\%

${ }^{2}$ Bacilo no fermentador

encontrarse de manera normal en el útero de alpacas con antecedentes reproductivos.

Serratia liquefaciens y Staphylococcus spp fueron hallados en muestras previas a la cópula en este estudio en animales del grupo de experiencia sexual comprobada (6.8 y $1.4 \%$, respectivamente; Cuadro 1$)$, pero no en las muestras posteriores a la cópula. No obstante, Serratia marcescens y Staphylococcus spp han sido aisladas en semen de jabalíes (Ubeda et al., 2013) y Serratia liquefaciens en prepucio de dromedarios (Serin et al., 2010), en tanto que
Staphylococcus epidermidis (28.1\%), Staphylococcus aureus y Staphylococcus intermedius (5.5\%) han sido hallados en semen de verracos (Pineda y Santander, 2007), y Staphylococcus spp (12.5\%) en semen congelado de toro (Morales et al., 2013). Asimismo, también se identificó a Micrococcus spp en muestras previas a la cópula (2.3\%), más no así luego de la cópula, a pesar de haberse observado Micrococcus sp en el prepucio de CSA (Jarvien y Kinyon, 2010) y en semen congelado de toro (16.7\%) (Morales et al., 2013). Por lo anteriormente expuesto sobre las bacterias aisladas 
Cuadro 2. Especies bacterianas aisladas de mucosa de úteros de alpacas sin experiencia sexual $(n=20)$ y con experiencia sexual $(n=40)$ colectadas a las 24 horas de la cópula

\begin{tabular}{lccccccc}
\hline Bacteria & \multicolumn{2}{c}{$\begin{array}{c}\text { Sin } \\
\text { experiencia }\end{array}$} & \multicolumn{2}{c}{$\begin{array}{c}\text { Con } \\
\text { experiencia }\end{array}$} & & Total & \\
\cline { 2 - 8 } & $\%$ & $\pm \mathrm{IC}$ & $\%$ & $\pm \mathrm{IC}$ & $\%$ & $\pm \mathrm{IC}^{1}$ & $\mathrm{n}=60$ \\
\hline Bacillus sp & 0 & 0 & 5 & 0.05 & 3.33 & 0.03 & 2 \\
Citrobacter feundii & 5 & 0.07 & 0 & 0.00 & 1.7 & 0.02 & 1 \\
Corynebacterium sp & 5 & 0.07 & 0 & 0.00 & 1.7 & 0.02 & 1 \\
Edwarsiella tarda & 5 & 0.07 & 0 & 0.00 & 1.7 & 0.02 & 1 \\
Enterobacter aerogenes & 10 & 0.10 & 2.5 & 0.04 & 5 & 0.04 & 3 \\
Enterobacter clocacae & 5 & 0.07 & 0 & 0.00 & 1.7 & 0.02 & 1 \\
Escherichia coli & 25 & 0.14 & 20 & 0.09 & 21.7 & 0.08 & 13 \\
Hafnia alvei & 5 & 0.07 & 10 & 0.07 & 8.3 & 0.05 & 5 \\
Klebsiella oxytoca & 15 & 0.11 & 0 & 0.00 & 5 & 0.04 & 3 \\
Morganella morganii & 5 & 0.07 & 0 & 0.00 & 1.7 & 0.02 & 1 \\
Proteus mirabilis & 10 & 0.10 & 0 & 0.00 & 3.3 & 0.03 & 2 \\
Proteus vulgaris & 5 & 0.07 & 0 & 0.00 & 1.7 & 0.02 & 1 \\
Providencia alcalifaciens & 10 & 0.10 & 2.5 & 0.04 & 5 & 0.04 & 3 \\
Providencia stuartii & 15 & 0.11 & 20 & 0.09 & 18.3 & 0.07 & 11 \\
Serratia rubidae & 0 & 0.00 & 7.5 & 0.06 & 5 & 0.04 & 3 \\
Shigella sonei & 5 & 0.07 & 2.5 & 0.04 & 3.3 & 0.03 & 2 \\
Shigella sp & 10 & 0.10 & 15 & 0.08 & 13.3 & 0.06 & 8 \\
Streptococcus sp & 0 & 0.00 & 5 & 0.05 & 3.3 & 0.03 & 2 \\
BNF ${ }^{2}$ & 0 & 0.00 & 2.5 & 0.04 & 1.7 & 0.02 & 1 \\
Levaduras & 10 & 0.10 & 10 & 0.07 & 10 & 0.06 & 6 \\
\hline
\end{tabular}

${ }^{1}$ Intervalo de confianza del $95 \%$

2 Bacilo no fermentador

precópula que no fueron aisladas en las muestras poscópula ( 24 horas después), y a pesar de que estas bacterias pueden ser transferidas por el macho; como ocurre en las yeguas (Alghamdi y Foster, 2005; Doty et al., 2011), es posible que haya sido debido a cambios en las condiciones intrauterinas promovidos por la liberación de citoquinas y al reclutamiento de polimorfonucleares afectando algunas bacterias; asimismo, cuando los espermatozoides migran a través del endometrio se libera $\mathrm{PGF}_{2 \alpha}$, lo cual causa contracciones uterinas, junto con la oxitocina (Madill et al., 2000).
Las bacterias que se hallaron en las muestras obtenidas luego de la cópula, tanto en los grupos con y sin experiencia sexual previa, fueron Klebsiella oxytoca (5\%), Morganella morganii (1.7\%), Proteus vulgaris $(1.7 \%)$ y Shigella sonei $(3.3 \%)$ (Cuadro 2); sin embargo, estas especies estuvieron ausentes en las muestras colectadas previo a la cópula (Cuadro 1). Algunos autores reportan a Morganella morganii $(3.8 \%)$ y Klebsiella oxytoca $(11.8 \%)$ en eyaculados de semen fresco de verraco (Ubeda et al., 2013), Klebsiella pneumoniae 
en semen fresco de verraco (Pineda y Santander, 2007); asimismo, Proteus mirabilis $(1.9 \%)$ en semen de verraco (Ubeda et al., 2013) y $P$. mirabilis y $P$. vulgaris en semen de verraco (Pineda y Santander, 2007) y en prepucio de CSA (Jarvien y Kinyon, 2010), lo que indicaría que el apareamiento sirve como fuente de contaminación y que podría contribuir a la posterior proliferación y establecimiento de infecciones uterinas.

Por último, el hallazgo de Hafnia alvei (6.1\%) y otras bacterias como Serratia liquefancies, Fusobacterium sp, Micrococcus sp, Streptococcus sp y bacilos no fermentadores en muy baja frecuencia ( 1 a 4 casos en 148 muestras), así como levaduras $(19.6 \%)$ antes de la cópula en el grupo de alpacas con experiencia sexual (Cuadro 1), se debe posiblemente a los antecedentes reproductivos de estos animales. La cópula y el parto son eventos que favorecen la colonización del lumen uterino (Sheldon, 2004; Foldi et al., 2006), dado el tipo de apareamiento agresivo que puede dañar la mucosa uterina (Tibary et al., 2001; Vaughan y Tibary, 2006), así como por la disminución del estado inmune durante el peri-parto, haciendo del útero un órgano vulnerable a infecciones ascendentes con bacterias oportunistas de la vagina y el medio ambiente (Sheldon et al., 2002; Singh et al., 2008). En este estudio, las hembras fueron montadas pocas semanas después del parto, lo cual puede haber favorecido el ingreso de las bacterias halladas.

\section{Conclusiones}

- Las bacterias más importantes y frecuentes en la mucosa uterina de las alpacas fueron Providencia stuartii (15.8 y $18.3 \%)$, Shigella sp (17.5 y 13.3\%), E. coli (26.0 y 21.7\%), Serratia rubidae (6.2 y 5.0\%), Hafnia alvei (5.1 y $8.3 \%$ ) y levaduras (16.4 y $10.0 \%)$ en muestras de colectadas antes y después de la cópula, respectivamente.
- Las hembras con experiencia sexual $(\mathrm{n}=148)$ presentaron Fusobacterium sp (1.4\%), Hafnia alvei (6.1\%), Micrococcus sp (2.7\%), Serratia liquefancies $(0.7 \%)$, Streptococcus sp (2.7\%), bacilos no fermentadores $(2.0 \%)$ y levaduras (19.6\%), mientras que estos gérmenes no se hallaron en alpacas sin experiencia sexual.

- Las bacterias encontradas únicamente posterior a la cópula fueron Klebsiella oxytoca (5\%), Morganella morganii (1.7\%), Proteus vulgaris $(1.7 \%) \mathrm{y}$ Shigella sonei (3.3\%).

\section{Literatura Citada}

1. Alghamdi AS, Foster DN. 2005. Seminal DNase frees spermatozoa entangled in neutrophil extracellular traps. Biol Reprod 73: 1174-81. doi: 10.1095/biolreprod.105.045666

2. Althouse GC, Kuster CE, Clark SG, Weisiger RM. 2000. Field investigations of bacterial contaminants and their effects on extended porcine semen. Theriogenology 53: 1167-1176. doi: 10.1016/S0093-691X(00)00261-2

3. Azawi OI, Taha MB. 2002. Clinical and bacteriological study of endometritis in Iraqi buffaloes. Iraqi J Vet Sci 16: 167-178.

4. Bravo P. 1994. Reproductive endocrinology of llamas and alpacas. Vet Clin North Am Food Anim Pract 10: 265-279. doi: 10.1016/S0749-0720(15)30561-2

5. Cárdenas N. 2013. Empadre y parición de alpacas. Cartilla técnica para la crianza mejorada de alpacas. Rome: FAO. [Internet]. Disponible en: http:// www.fao.org/family-farming/detail/es/c/ 369918/

6. Doty A, Buhi WC, Benson S, Scoggin KE, Pozor M, Macpherson M, Mutz M, Troedsson MH. 2011. Equine CRISP3 modulates interaction between spermatozoa and polymorphonuclear neutrophils. Biol Reprod 85: 157-64. doi: 10.1095/biolreprod.110.084491 
7. Enany M, Hanafi MS, El-Ged AG, ElSeedy FR, Khalid A. 1990. Microbiological studies on endometritis in she-camels in Egypt. J Egypt Vet Med Assoc 50: 229-243.

8. [FAO] Organización de las Naciones Unidas para la Agricultura y la Alimentación. 2005. Situacion actual de los camelidos sudamericanos en el Perú. [Internet]. Disponible en: http:// tarwi.lamolina.edu.pe/ emellisho/ zootecnia_archivos/situacion $\%$ 20alpcas\%20peru.pdf

9. Fernández A, Dimoso Z. 1984. Estudio cualitativo y cuantitativo de la flora bacteriológica de las secreciones cervicouterinas de vacas clínicamente sanas. En: IV Conferencia de Ciencias Agropecuarias. Cuba.

10. Fernández A, Villavicencio L, Peláez $R$, Silveira E, Garcia P, Peraza N. 1984. Estudio cualitativo y cuantitativo de la microflora de secreciones cervicouterinas en vacas con repetición de celo. Rev Cub Reprod Anim 10: 83-93.

11. Foldi J, Kulcsar M, Pecsi A, Huyghe $B$, de Sa C, Lohuis JA, Cox P, Huszenicza G 2006. Bacterial complications of postpartum uterine involution in cattle. Anim Reprod Sci 96: 265-281. doi: 10.1016/j.anireprosci. 2006.08.006

12. Ghanem M, Shalaby AH, Sharawy $S$, Saleh N. 2002. Factors leading to endometritis in Egypt with special reference to reproductive performance. J Reprod Dev 48: 371-375. doi: 10.1262/ jrd.48.371

13. Ghoneim IM, Waheed MM, Al-Hofofi AN, Fayez MM, Al-Eknah MM, AlBusadah KA, Al-Humam NA. 2014. Evaluation of the microbial quality of fresh ejaculates of camel (Camelus dromedarius) semen. Anim Reprod Sci 149: 218-223. doi: 10.1016/j.anireprosci.2014.07.021

14. [INEI] Insitituto Nacional de Estadística e Informática. 2012. IV Censo Nacional Agropecuario. [Internet]. Disponible en: http://censos.inei.gob.pe/ cenagro/tabulados/
15. Jang SS, Hirsh DC. 1994. Characterization, distribution, and microbiological associations of Fusobacterium spp in clinical specimens of animal origin. J Clin Microbiol 32: 384-387.

16. Jarvien J, Kinyon J. 2010. Preputial microflora of llamas (Lama glama) and alpacas (Vicugna pacos). Small Ruminn Res 90: 156-160. doi: 10.1016/ j.smallrumres.2010.01.007

17. Koneman E, Winn W, Allen S, Janda $W$, Procop G, Schreckenberger P, et al. 2006. Diagnóstico microbiológico. Buenos Aires: Ed Médica Panamericana. $1691 \mathrm{p}$.

18. Madill S, Troedsson MH, Alexander SL, Shand N, Santschi EM, Irvine CH. 2000. Simultaneous recording of pituitary oxytocin secretion and myometrial activity in oestrous mares exposed to various breeding stimuli. J Reprod Fertil 56: 351-361.

19. Morales S, Cabrera P, Pantoja C, García D, Solis N. 2013. Evaluación de la carga bacteriana en pajillas de semen congelado de toros del Banco Nacional de Semen. Científica 10: 28-36.

20. Mshelia GD, Okpaje G, Voltaire YA, Egwu GO. 2014. Comparative studies on genital infections and antimicrobial susceptibility patterns of isolates from camels (Camelus dromedarius) and cows (Bos indicus) in Maiduguri, northeastern Nigeria. SpringerPlus 3: 91. doi: 10.1186/2193-1801-3-91

21. [OIE] Organización Mundial de Salud Animal. 2008. Manual de la OIE sobre animales terrestres. Cap 2.9.9. Salmonelosis. En: Manual de las pruebas de diagnóstico y de las vacunas para los animales terrestres. $6^{\mathrm{a}}$ ed. Paris: OIE. p 1268-1286. [Internet]. Disponible en: http://www.oie.int/fileadmin/Home/eng/ Health_standards/tahm/2008/pdf/ 2.09.09 salmonellosis.pdf

22. Pineda Y, Santander J. 2007. Evaluación de la flora bacteriana del semen de verracos en granjas porcinas de Venezuela. Zootecnia Trop 25: 173-177. 
23. Platt H, Atherston JG, Orskov I. 1977. Klebsiella and enterobacter organisms isolated from horses. J Hyg Camb 77: 401-408.

24. Ricketts SW. 1981. Bacterioloical examinations of the mare's cervix: techniques and interpretation of results. Vet Rec 108: 46-51.

25. Serin I, Ceylan A, Kirkan S, Parin U. 2010. Preputial bacterial flora and antibiotic susceptibility in wrestling dromedary bulls in Aydin region of Turkey. J Anim Vet Adv 9: 482-485. doi: 10.3923/javaa.2010.482.485

26. Sheldon IM. 2004. The postpartum uterus. Vet Clin North Am Food Anim Pract 20: 569-591 doi: 10.1016/ j.cvfa.2004.06.008

27. Sheldon IM, Noakes DE, Rycroft AN, Pfeiffer DU, Dobson H. 2002. Influence of uterine bacterial contamination after parturition on ovarian dominant follicle selection and follicle growth and function in cattle. Reproduction 123: 837-845.

28. Singh J, Murray RD, Mshelia G, Woldehiwet Z. 2008. The immune status of the bovine uterus during the peripartum period. Vet J 175: 301-309. doi: 10.1016/j.tvj1.2007.02.003

29. Tibary A. 2004. Infertility in female camelid 2: causes and treatment. In: Large Animal Proc North American Veterinary Conference 18: 287-289.

30. Tibary A, Anouassi A, Memon MA. 2001. An approach to the diagnosis of infertility in camelids: retrospective study in alpaca, llamas and camels. J Camel Pract Res 8: 167-179.

31. Tibary A, Fite C, Anouassi A, Sghiri $A$. 2006. Infectious causes of reproductive loss in camelids. Theriogenology 66: 633-647. doi: 10.1016/j.theriogenology.2006.04.008

32. Ubeda JL, Ausejo R, Dahmani Y, Falceto MV, Usan A, Malo C, PerezMartinez FC. 2013. Adverse effects of members of the Enterobacteriaceae family on boar sperm quality. Theriogenology 80: 565-570. doi: 10.1016/ j.theriogenology.2013.05.022

33. Vaughan JL, Tibary A. 2006. Reproduction in female South American camelids: a review and clinical observations. Small Rumin Res 61: 259-281. doi: 10.1016/j.smallrumres. 2005.07.015 